KUNS-1630

hep-th/0001161

\title{
Space-time uncertainty relation and Lorentz invariance
}

\author{
Naoki SASAKURA* \\ Department of Physics, Kyoto University, Kyoto 606-8502, Japan
}

January, 2000

\begin{abstract}
We discuss a Lorentz covariant space-time uncertainty relation, which agrees with that of Karolyhazy-Ng-van Dam when an observational time period $\delta t$ is larger than the Planck time $l_{P}$. At $\delta t \lesssim l_{P}$, this uncertainty relation takes roughly the form $\delta t \delta x \gtrsim l_{P}^{2}$, which can be derived from the condition prohibiting the multi-production of probes to a geometry. We show that there exists a minimal area rather than a minimal length in the four-dimensional case. We study also a three-dimensional free field theory on a non-commutative space-time realizing the uncertainty relation. We derive the algebra among the coordinate and momentum operators and define a positive-definite norm of the representation space. In fourdimensional space-time, the Jacobi identity should be violated in the algebraic representation of the uncertainty relation.
\end{abstract}

*sasakura@gauge.scphys.kyoto-u.ac.jp 


\section{Introduction}

There is a suspicion that the space-time is not a smooth manifold in a small scale as assumed in general relativity, but should be replaced by a new quantum geometry [1]. Uncertainty relation of space-time gives an approach to such quantum space-time. Since the uncertainty relation of quantum mechanics can be obtained with a simple thought experiment, we may expect that the space-time uncertainty relation might be guessed without knowing the complete theory of quantum gravity. In fact there are several proposals of the uncertainty relation of spacetime. Some come from the analysis of the scattering and the fluctuation sizes of strings and branes in string theory [2, 3, , 4, 5]. Some others are derived from the investigations of the thought measuring process of space-time distances or locations, using only the knowledge of the quantum mechanics and the general relativity [6, 7, 8, 9, 10]. The main idea of the space-time uncertainty relation obtained from such a thought measuring process is as follows. To measure the space-time distances or locations precisely, we need a high energy probe to minimize the space-time size of the wave function of the probe. But, on the other hand, since gravity couples to the energy-momentum tensor, the high energy probe would generate large fluctuations of metric. Since the location of the probe is spread quantum mechanically, the metric fluctuations have indeterminable components. Thus there exists limitation of precise measurement of space-time distances or locations.

The quantum uncertainty of measuring a space-time distance $l, \delta l \gtrsim\left(l_{P}^{2} l\right)^{1 / 3}$, was obtained first by Karolyhazy [7], and also by Ng and van Dam [8] in a thought experiment first proposed by Salecker and Wigner [6]. Amelino-Camelia used the uncertainty relation in the form $\delta l \gtrsim$ $\left(l_{P}^{2} \delta t\right)^{1 / 3}$ with an observational time period $\delta t$, and pointed out the possibility of observing this quantum fluctuation of the geometry with a gravitational-wave interferometer [11. (1) Barrow argued that this uncertainty relation gives qualitatively the same prediction of the life-time of a black hole [14] as that derived from Hawking radiation.

In our previous paper [15], we used this uncertainty relation in the form $\delta V \gtrsim l_{P}^{2} \delta t$, where $\delta V$ denotes an uncertainty of a spatial volume. In this case, the uncertainty relation is applied to a four-dimensional space-time volume. This interpretation lead to an entropy bound $S \lesssim \sqrt{E V} / l_{P}$, where $E$ and $V$ are the total energy and volume of a system, respectively. We pointed out that this entropy bound fits well to the holographic principle proposed by 't Hooft and Susskind [16]. In fact, a similar kind of entropy bound was assumed in order to prove

\footnotetext{
${ }^{1}$ See [12] for criticism against this idea, and also [13] for protection
} 
rigorously [17] the Bousso's covariant entropy bound [18]. Recently, a generally covariant formula of a similar entropy bound was proposed by Brustein and Veneziano [19], generalizing the Hubble-entropy-bound [20]. Thus it would be a natural expectation that the space-time uncertainty relation can also be formulated in a generally covariant form.

In this paper, we discuss a Lorentz covariant formulation of the space-time uncertainty relation above, as the smallest step toward a generally covariant formulation. We first point out that the condition that the multi-production of probe particles should not occur in the measurement process leads to the uncertainty relation $\delta t \delta x \gtrsim l_{P}^{2}$ at $\delta t \lesssim l_{P}$. This relation and $\delta V \gtrsim l_{P}^{2} \delta t$ at $\delta t \gtrsim l_{P}$ can be naturally combined into the form $l_{P}^{2}\left|\delta x_{i_{1}}^{\mu} n_{\mu}\right| \lesssim$ $\left|\varepsilon_{\mu \nu \rho \sigma} n^{\mu} \delta x_{i_{2}}^{\nu} \delta x_{i_{3}}^{\rho} \delta x_{i_{4}}^{\sigma}\right|$ for any observer $n^{\mu}$. In general dimensions (larger than two), the uncertainty relation is just obtained by a straightforward generalization. With this motivation, we investigate a free field theory on a non-commutative three-dimensional space-time with the coordinate commutation relations of Lie algebra so(2,1). We construct the momentum operators on this non-commutative space-time and define a positive-definite norm on which the coordinate and momentum operators are hermite. We study the Klein-Gordon equation on this non-commutative space-time. We found a two-fold degeneracy of the spectra. Lastly we point out that, in the case of the four-dimensional space-time, the Jacobi identity should be violated in algebraic representations of the uncertainty relation.

\section{Uncertainty relation of space-time}

The metric tensor field in the general relativity could be measured by following the trajectory of a point particle. In classical mechanics, the probe particle can be regarded as a point, hence we obtain a smooth manifold, which is assumed in the general relativity. However, in quantum mechanics, since the probe particle is spread over its wave function, the measurement becomes necessarily vague. We shall discuss this measurement uncertainty in a flat Minkowski space-time. To be self-contained, we begin with the derivations of the space-time uncertainty relation of Karolyhazy-Ng-van Dam [7, 8] with a new ingredient.

We follow, for a time period $\delta t$, a probe particle with a mass $m$ which has initially a vanishing total momentum and the gaussian wave packet with $\left\langle(\delta x)^{2}\right\rangle_{t=0}=\sigma^{2}$. We assume the wave function is symmetric under the spatial rotation. In non-relativistic approximation, the distribution of the gaussian wave packet becomes $\left\langle(\delta x)^{2}\right\rangle_{t=\delta t}=\sigma^{2}+(\delta t)^{2} / 4 m^{2} \sigma^{2}$ after the time period $\delta t$, by solving the Shrödinger equation. This second term comes essentially 
from the momentum distribution, which is inversely proportional to the spatial distribution $\sigma$. This shows that, even if we have a smaller wave packet initially, we do not necessarily have a smaller wave packet for the whole period of the measuring process. Rather it has a finite minimum

$$
\sigma_{\min } \equiv \operatorname{Min}_{\sigma} \sqrt{\sigma^{2}+(\delta t)^{2} / 4 m^{2} \sigma^{2}} .
$$

Without taking into account the condition discussed in the following paragraph, the minimum value is $\sqrt{\delta t / m}$ at $\sigma=\sqrt{\delta t / 2 m}$. At this minimum, the momentum distribution is in the order of $\delta p \sim 1 / \sigma \sim \sqrt{m / \delta t}$. Hence the condition for the non-relativistic approximation, $\delta p \lesssim m$, to hold is equivalent to $m \gtrsim 1 / \delta t$. In fact, this condition must be imposed from the beginning, since, if not, the multi-production of the probes will occur because of the quantum mechanical uncertainty relation between energy and time, and the measurement by a particle probe must be abandoned. For a smaller $\sigma$, we should treat in a fully relativistic way. But the size of the wave packet $\left\langle(\delta x)^{2}\right\rangle_{t=\delta t}$ in this parameter region will be anyway larger than the minimum value $\sigma_{\min }$. Thus the non-relativistic approximation is enough to obtain $\sigma_{\min }$ if $m$ is not so near to $1 / \delta t$.

The other ingredient of the Karolyhazy-Ng-van Dam type uncertainty relation is the condition that the probe should not become a black hole for it to be followed. This condition is hard to evaluate in a reliable manner, since we need a quantum field theory of general relativity to do so. We could approximate the condition with that the mean mass distribution $\rho=m|\varphi(x)|^{2}$, where $\varphi(x)$ is the gaussian wave function with distribution $\sigma$, does not make a black hole in the classical general relativity, just believing that the result is qualitatively correct. This condition is given by 21]

$$
2 m G \int_{|x|<R} d^{3} x|\varphi(x)|^{2}<R \quad \text { for all } R,
$$

where $G$ is the gravitational constant. This gives

$$
\sigma \gtrsim l_{P}^{2} m
$$

which agrees with the intuition that the wave packet cannot be confined in the size of the Schwarzschild radius of the probe particle. Thus, from (1) and (3), we finally obtain the shortest observable spatial length in an observation with a time period $\delta t$ :

$$
\delta x_{\text {min }}=\operatorname{Min}_{m \gtrsim \frac{1}{\delta t}} \operatorname{Min}_{\sigma \gtrsim l_{P}^{2} m} \sqrt{\sigma^{2}+(\delta t)^{2} / 4 m^{2} \sigma^{2}},
$$

where we take into account the condition $m \gtrsim \frac{1}{\delta t}$, which prohibits the multi-production of the probe particles. This condition is a new ingredient of this paper. When $\delta t \gtrsim l_{P}$, the 
minimum of (幽) is at $\sigma=l_{P}^{2} m, m^{6}=(\delta t)^{2} / 2 l_{P}^{8}$. Thus we obtain the Karolyhazy-Ng-van Dam type uncertainty relation $[7,8,11]$ :

$$
(\delta x)^{3} \gtrsim\left(\delta x_{\min }\right)^{3} \sim l_{P}^{2} \delta t .
$$

The non-relativistic approximation is valid for this case, since $m / \delta p \sim \sigma m \sim\left(\delta t / l_{P}\right)^{2 / 3} \gtrsim 1$ at the minimum. When $\delta t \lesssim l_{P}$, the minimum is at $\sigma=l_{P}^{2} m, m=1 / \delta t$. Thus we obtain

$$
\delta x \gtrsim \delta x_{\min } \sim \frac{l_{P}^{2}}{\delta t} .
$$

Again the non-relativistic approximation is valid, since $m / \delta p \sim \sigma m \sim\left(l_{P} / \delta t\right)^{2} \gtrsim 1$ at the minimum. Form the inequalities (5) and (6), one founds that the Planck length is the minimal spatial length, $\delta x \gtrsim l_{P}$, while $\delta t$ is not bounded. This rather odd result will be remedied properly in the Lorentz covariant formulation discussed below.

The relations (5) and (6) can be rewritten in the following suggestive ways:

$$
\begin{aligned}
(\delta x)^{3} & \gtrsim l_{P}^{2} \delta t \\
(\delta x)^{2} \delta t & \gtrsim l_{P}^{2} \delta x
\end{aligned}
$$

Thus we propose that the uncertainty relation of space-time has the following Lorentz covariant form:

$$
l_{P}^{2} \delta x_{i_{1}}^{\mu} n_{\mu} \lesssim\left|\varepsilon_{\mu \nu \rho \sigma} n^{\mu} \delta x_{i_{2}}^{\nu} \delta x_{i_{3}}^{\rho} \delta x_{i_{4}}^{\sigma}\right| \text { for any } n^{\mu}
$$

where $\delta x_{i}^{\mu}(i=1,2,3,4)$ are the four vectors defining a space-time volume, and the inequality (8) should be satisfied for all ordering of $\delta x_{i}^{\mu}$. The case $\delta x_{1}=(\delta t, 0,0,0), \delta x_{2}=$ $(0, \delta x, 0,0), \delta x_{3}=(0,0, \delta x, 0), \delta x_{4}=(0,0,0, \delta x)$ in (8) reproduces (7). By substituting $n^{\mu}=$ $\delta x_{i}^{\mu}$ in (8), we see that the four vectors $\delta x_{i}^{\mu}$ must be orthogonal among each other.

The condition (8) might be too strong. Physically, the vector $n^{\mu}$ would denote the velocity vector of an observer. In fact, the following weaker condition is enough to reproduce (7):

$$
l_{P}^{2}\left|\delta x_{i_{1}}^{\mu} n_{\mu}\right| \lesssim\left|\varepsilon_{\mu \nu \rho \sigma} n^{\mu} \delta x_{i_{2}}^{\nu} \delta x_{i_{3}}^{\rho} \delta x_{i_{4}}^{\sigma}\right| \text { for any time-like } n^{\mu}
$$

There would be other possible Lorentz covariant formulations which reproduce (7). Presently, we do not have any criteria to choose one of them.

Now let us consider the case $\delta x_{1}=(\delta t, 0,0,0), \delta x_{2}=\left(0, \delta l_{1}, 0,0\right), \delta x_{3}=\left(0,0, \delta l_{2}, 0\right), \delta x_{4}=$ $\left(0,0,0, \delta l_{3}\right)$. From (8) or (9), we obtain

$$
l_{P}^{2} \delta t \lesssim \delta l_{1} \delta l_{2} \delta l_{3}
$$




$$
\begin{aligned}
l_{P}^{2} \delta l_{1} & \lesssim \delta l_{2} \delta l_{3} \delta t \\
l_{P}^{2} \delta l_{2} & \lesssim \delta l_{3} \delta l_{1} \delta t \\
l_{P}^{2} \delta l_{3} & \lesssim \delta l_{1} \delta l_{2} \delta t
\end{aligned}
$$

From the first and the second equation, we find

$$
\delta l_{2} \delta l_{3} \gtrsim \frac{1}{2} l_{P}^{2}\left(\frac{\delta l_{1}}{\delta t}+\frac{\delta t}{\delta l_{1}}\right) \gtrsim l_{P}^{2} .
$$

Similarly, we obtain

$$
\begin{gathered}
\delta l_{1} \delta l_{2} \gtrsim l_{P}^{2}, \\
\vdots
\end{gathered}
$$

Thus we conclude that the area is bounded from below by the square of the Planck length, while the length is not in general. We have found a minimal area rather than a minimal length in four dimensional space-time.

The generalization of (8) and (9) to any space-time dimension is obvious. Let us consider a three-dimensional case with $\delta x_{1}=(\delta t, 0,0), \delta x_{2}=\left(0, \delta l_{1}, 0\right), \delta x_{3}=\left(0,0, \delta l_{2}\right)$. The uncertainty relation leads to

$$
\begin{aligned}
& l_{P} \delta t \lesssim \delta l_{1} \delta l_{2}, \\
& l_{P} \delta l_{1} \lesssim \delta l_{2} \delta t \\
& l_{P} \delta l_{2} \lesssim \delta l_{1} \delta t
\end{aligned}
$$

From these inequalities, we obtain $\delta t, \delta l_{1}, \delta l_{2} \gtrsim l_{P}$. Thus there is a minimal length in threedimensional space-time.

\section{Algebraic representation}

In this section, we shall discuss an algebraic representation of the uncertainty relation (13) in three-dimensional space-time in the spirit of Snyder [22], and study a free field theory on a non-commutative space-time obtained from it. Lastly we give some comments for the four-dimensional case.

The algebra we use for the coordinates is the Lie algebra $s o(2,1)$ :

$$
\left[x^{\mu}, x^{\nu}\right]=i l_{P} \varepsilon^{\mu \nu \rho} x_{\rho} .
$$


The motivation to use this algebra is that, if there exists a semi-positive definite norm on the representation space of $x^{\mu}$ with an appropriate $\dagger$ operation, we can show that

$$
\begin{aligned}
& l_{P}^{2}\left\langle x^{0}\right\rangle^{2} \leq 4\left\langle\left(x^{1}\right)^{2}\right\rangle\left\langle\left(x^{2}\right)^{2}\right\rangle \\
& \quad \vdots \quad
\end{aligned}
$$

by using $\left\langle\left(x^{1}+i \lambda x^{2}\right)^{\dagger}\left(x^{1}+i \lambda x^{2}\right)\right\rangle \geq 0$ and so on for any $\lambda$. The inequalities (15) may be regarded as a realization of the space-time uncertainty relation (13).

Firstly we shall discuss the momentum operator on this space-time. Let us assume the following form of the commutation relations for the momentum operators:

$$
\begin{aligned}
{\left[p^{\mu}, p^{\nu}\right] } & =0 \\
{\left[p^{\mu}, x^{\nu}\right] } & =-i \eta^{\mu \nu} f\left(l_{P}^{2} p^{2}\right)+i l_{P} \varepsilon^{\mu \nu \rho} p_{\rho} g\left(l_{P}^{2} p^{2}\right),
\end{aligned}
$$

where $f, g$ are some functions with $f(0)=1$ so that we recover the usual commutation relations in the limit $l_{P} \rightarrow 0$. In (16), we have ignored a term with the tensor structure $p^{\mu} p^{\nu}$, because this term could be absorbed by the redefinition $p^{\mu} \rightarrow p^{\mu} k\left(l_{P}^{2} p^{2}\right)$. We take the signature $\eta^{\mu \nu}=(-1,1,1)$ and $\varepsilon^{012}=1$. The criteria to determine these functions $f, g$ are the Jacobi identities. For the cases $[x,[x, x]+\cdots,[x,[p, p]]+\cdots$ and $[p,[p, p]]+\cdots$, the Jacobi identities are satisfied obviously. In the remaining case, we have

$$
\begin{aligned}
{\left[p^{\mu},[\right.} & \left.\left.x^{\nu}, x^{\rho}\right]\right]+\left[x^{\rho},\left[p^{\mu}, x^{\nu}\right]\right]+\left[x^{\nu},\left[x^{\rho}, p^{\mu}\right]\right] \\
= & l_{P} \varepsilon^{\mu \nu \rho} f(1-2 g)+l_{P}^{2}\left(\eta^{\mu \nu} p^{\rho}-\eta^{\mu \rho} p^{\nu}\right)\left(2 f f^{\prime}-g+g^{2}\right) \\
& +2 l_{P}^{3}\left(\varepsilon^{\mu \rho \sigma} p_{\sigma} p^{\nu}-\varepsilon^{\mu \nu \sigma} p_{\sigma} p^{\rho}\right) g^{\prime} f .
\end{aligned}
$$

For this to vanish, $f$ and $g$ are determined as

$$
f(u)=\sqrt{1+\frac{1}{4} u}, \quad g(u)=\frac{1}{2} .
$$

The algebra (16) and (18) we have obtained has a great similarity with that discussed by Maggiore [23]. From (16) and (18), we immediately obtain

$$
\Delta x^{\mu} \Delta p^{\mu} \gtrsim \frac{1}{2}\left\langle\sqrt{1+\frac{l_{P}^{2} p^{2}}{4}}\right\rangle,
$$

where the summation over $\mu$ is not assumed. As was discussed by Maggiore, this uncertainty relation (19) has the following interesting features. In the high Euclidean momentum regime with $\left\langle p^{i}\right\rangle^{2} \sim\left(\Delta p^{i}\right)^{2} \gg l_{P}^{2}$, we get

$$
\Delta x^{i} \gtrsim \frac{l_{P}}{4}
$$


Thus we obtain a minimal spatial length. However, since, in our Lorentzian case, the righthand side of (19) can take a fixed value by canceling a spatial momentum with its timecomponent, we do not argue that there exists a universal minimal length in the sense discussed in [24]. In the regime $\left|\left\langle p^{2}\right\rangle\right| \ll l_{P}^{-2}$, we obtain an uncertainty relation similar to that from string theory [2, [3]:

$$
\Delta x^{\mu} \Delta p^{\mu} \gtrsim \frac{1}{2}+\frac{l_{P}^{2}\left\langle p^{2}\right\rangle}{16} .
$$

Now we shall discuss an explicit representation of the operators which satisfy the commutation relations (14) and (16) with (18). Since the momentum operators are commutative, we work in the representation in which the momentum operators are diagonal. For $x^{\mu}$ to satisfy (14) and (16) with (18), we easily obtain

$$
x^{\mu}=-\frac{i l_{P}}{2} \varepsilon^{\mu \nu \rho} p_{\nu} \frac{\partial}{\partial p^{\rho}}+i \sqrt{1+\frac{l_{P}^{2} p^{2}}{4}} \frac{\partial}{\partial p_{\mu}}+l_{P}^{2} A\left(l_{P}^{2} p^{2}\right) p^{\mu},
$$

where $A\left(l_{P}^{2} p^{2}\right)$ is an arbitrary function of $p^{2}$. The measure of the representation space is also determined uniquely up to a multiplication constant under the condition that the operators $x^{\mu}$ and $p^{\mu}$ be hermite. It is

$$
\left\langle\Psi_{1}|\mathcal{O}(x, p)| \Psi_{2}\right\rangle=\int d^{3} p\left(1+\frac{l_{P}^{2} p^{2}}{4}\right)^{-\frac{1}{2}} \Psi_{1}^{*}(p) \mathcal{O}(x, p) \Psi_{2}(p) .
$$

For $x^{\mu}$ to be hermite, the arbitrary function $A\left(l_{P}^{2} p^{2}\right)$ of (22) must be a real function.

The measure (23) sets bounds on the range of the momentum $p^{\mu}$ :

$$
-p^{2}<\frac{4}{l_{P}^{2}}
$$

Thus there exists a mass upper-bound. From the explicit representation (22) of $x^{\mu}$, we can see that the operation $x^{\mu}$ does not violate this bound. This is obvious for the first and third terms of (22). The second term changes $p^{2}$ in general, but, at the boundary $p^{2}=-\frac{4}{l_{P}^{2}}$, the change vanishes. However, as we will see soon, there is a little complication concerning the global structure of the representation space, and we will find that the momentum representation space is in fact two-fold degenerate.

Before discussing this feature, let us look for a wave function $\Psi$ which satisfies the KleinGordon equation, $\left(p^{\mu} p_{\mu}+m^{2}\right) \Psi=0$. The solution is trivially given by $\Psi_{ \pm}(p)=\theta\left( \pm p^{0}\right) \delta\left(p^{2}+\right.$ $\left.m^{2}\right)$ in the momentum representation. There is also another route to obtain the wave function in terms of the coordinates $x^{\mu}$. Let us assume the following form of the wave function:

$$
\Psi\left(k^{\mu}\right)=e^{i k^{\mu} x_{\mu}}|0\rangle,
$$


where $k^{\mu}$ are $c$-numbers, and $|0\rangle$ denotes the momentum zero eigenstate $p^{\mu}|0\rangle=0$. Now we operate $p^{\mu}$ on this state:

$$
\begin{aligned}
p^{\mu} e^{i k^{\mu} x_{\mu}}|0\rangle & =\int_{0}^{1} d s e^{i(1-s) k^{\mu} x_{\mu}}\left[p^{\mu}, i k^{\nu} x_{\nu}\right] e^{i s k^{\mu} x_{\mu}}|0\rangle \\
& =\int_{0}^{1} d s e^{i(1-s) k^{\mu} x_{\mu}}\left(k^{\mu} \sqrt{1+\frac{1}{4} l_{P}^{2} p^{2}}-\frac{1}{2} l_{P} \varepsilon^{\mu \nu \rho} k_{\nu} p_{\rho}\right) e^{i s k^{\mu} x_{\mu}}|0\rangle
\end{aligned}
$$

where we have used the formula $\frac{d}{d t} e^{A}=\int_{0}^{1} d s e^{(1-s) A} \frac{d A}{d t} e^{s A}$ and the commutation relations (16). Repeating the above operation of $p^{\mu}$ perturbatively in $l_{P}$, one can see that the wave function $\Psi\left(k^{\mu}\right)$ is in fact an eigenstate of the momentum operator $p^{\mu}$ :

$$
p^{\mu} e^{i k^{\mu} x_{\mu}}|0\rangle=k^{\mu} h\left(k^{2}\right) e^{i k^{\mu} x_{\mu}}|0\rangle .
$$

To determine the function $h(u)$, we substitute (27) into (26). Then we obtain an integration equation,

$$
h(u)=\int_{0}^{1} d s \sqrt{1+\frac{1}{4} l_{P}^{2}\left(h\left(s^{2} u\right)\right)^{2} s^{2} u} .
$$

By taking the derivative with respect to $u$, we obtain a differential equation

$$
2 u h^{\prime}+h-\sqrt{1+\frac{1}{4} l_{P}^{2} u h^{2}}=0 .
$$

The solution of this equation is given by

$$
\begin{aligned}
h\left(k^{2}\right) & =\frac{2}{l_{P}} \frac{1}{\sqrt{k^{2}}} \sinh \left(\frac{l_{P}}{2} \sqrt{k^{2}}\right) \text { for a space-like } k^{2} \\
h\left(k^{2}\right) & =\frac{2}{l_{P}} \frac{1}{\sqrt{-k^{2}}} \sin \left(\frac{l_{P}}{2} \sqrt{-k^{2}}\right) \text { for a time-like } k^{2}
\end{aligned}
$$

Thus the field equation becomes

$$
\left(p^{\mu} p_{\mu}+m^{2}\right) \Psi\left(k^{\mu}\right)=\left(-\frac{4}{l_{P}^{2}} \sin ^{2}\left(\frac{l_{P}}{2} \sqrt{-k^{2}}\right)+m^{2}\right) \Psi\left(k^{\mu}\right)=0 .
$$

In the case $m=0$, the solutions for (31) are:

$$
k^{2}=-\left(\frac{2 \pi n}{l_{P}}\right)^{2}
$$

with integer $n$. Thus it looks apparently as if there are an infinite number of additional states.

To study this issue in more detail, let us consider the operator $\Omega=e^{2 \pi i x^{0} / l_{P}}$. The following discussion is obviously applicable also to the Lorentz transform of $\Omega$. Th e operator $\Omega$ 
commutes with the coordinate $x^{\mu}$, because it is just the $2 \pi$ rotation in the spatial plane. To see the operation on the momentum $p^{\mu}$, let us consider the operator $\Omega(s)=e^{i s x^{0} / l_{P}}$ with an arbitrary real number $s$, and define $p^{\mu}(s)=\Omega(s) p^{\mu} \Omega(-s)$. Then we have

$$
\begin{aligned}
\frac{d p^{\mu}(s)}{d s} & =\frac{i}{l_{P}}\left[x^{0}, p^{\mu}(s)\right] \\
& =\left\{\begin{array}{l}
\frac{1}{l_{P}} \sqrt{1+\frac{l_{P}^{2} p^{2}(s)}{4}} \text { for } \mu=0, \\
\frac{1}{2} \varepsilon^{i j} p_{j}(s) \text { for } \mu=i,
\end{array}\right.
\end{aligned}
$$

where $i, j$ denote the indices for the two-dimensional spatial plane, and $\varepsilon^{i j}$ is the antisymmetric tensor with $\varepsilon^{12}=1$. The differential equation (33) is easily solved with the parameterization $p^{0}(s)=\left(2 / l_{P}\right) \sqrt{1+l_{P}^{2} a^{2} / 4} \sin \theta, p^{1}(s)=a \cos \varphi, p^{2}(s)=a \sin \varphi:$

$$
\theta-\theta_{0}=\varphi-\varphi_{0}=-\frac{1}{2} s
$$

where $\theta_{0}$ and $\varphi_{0}$ are the initial values. Thus $\Omega=\Omega(2 \pi)$ is a PT transform of the states. By performing the operation $\Omega$ twice, a state will be transformed to the identical state. However, as we will explain in the followings, a state obtained by operating $\Omega$ once is not identical with the anti-particle state of the original state. In the derivation of (34), we assumed that the square root in (33) or (18) can take both positive and negative values: $\sqrt{1+l_{P}^{2} p^{2}(s) / 4}=$ $\sqrt{1+a^{2} l_{P}^{2} / 4} \cos \theta$. In other words, there are two patches where the square root takes positive or negative values. Thus it is more appropriate to parameterize the representation space in terms of $\theta$ and $p^{i}$ with $p^{0}=\left(2 / l_{P}\right) \sqrt{1+\left(p^{i}\right)^{2} l_{P}^{2} / 4} \sin \theta$, where $\theta$ has the range $0 \leq \theta<2 \pi$ with the identification between $\theta=0$ and $\theta=2 \pi$. With this parameterization, the measure becomes

$$
\int d^{3} p\left(1+p^{2} l_{P}^{2} / 4\right)^{-\frac{1}{2}}=\frac{2}{l_{P}} \int d \theta d^{2} p .
$$

Thus we have obtained safely a positive definite norm. Since, for a given value of momentum, we have two choices for $\theta$ (unless $\theta= \pm \pi / 2$ ), we conclude that the momentum representation space as well as the spectra of the states satisfying the Klein-Gordon equation are two-fold degenerate.

In (14), the translational symmetry is not manifest. As was discussed in [22], the notion of translational symmetry should be appropriately substituted for the non-commutative spacetime. The simplest candidate for the translation by a c-number vector $v^{\mu}$ is the multiplication of $e^{-i v^{\mu} p_{\mu}}$ on a wave function $\Psi\left(p^{\mu}\right)$ in the momentum representation. In fact, from (22), we obtain $e^{i v^{\mu} p_{\mu}} x^{\mu} e^{-i v^{\mu} p_{\mu}}=x^{\mu}+k^{\mu}$ in the limit $l_{P} \rightarrow 0$. The whole theory should respect this symmetry. 
Finally let us discuss whether it is possible to represent the uncertainty relation algebraically in four dimensions as in the three-dimensional case. The uncertainty relation has roughly the form $\varepsilon_{\mu \nu \rho \sigma} \delta x^{\mu} \delta x^{\nu} \delta x^{\rho} \gtrsim l_{P}^{2} \delta x_{\sigma}$. We now assume the greater side is represented as a commutation of operators. Moving one of the coordinate operator from the left to the right-hand side as the momentum operator, a possibility is

$$
\left[x^{\mu}, x^{\nu}\right]=i l_{P}^{2} \varepsilon^{\mu \nu \rho \sigma} p_{\rho} x_{\sigma}+o\left(l_{P}^{2}\right) .
$$

This algebra has a similarity with the Snyder algebra [22]. The Snyder algebra has the form $\left[x^{\mu}, x^{\nu}\right]=i a^{2} J^{\mu \nu}$, where $a$ and $J^{\mu \nu}$ denote the length scale and the Lorentz transformation generators associated to the non-commutative space-time, respectively. The difference is that, in our case (36), the right-hand side is twisted:

$$
\left[x^{\mu}, x^{\nu}\right] \sim i l_{P}^{2} \varepsilon^{\mu \nu \rho \sigma} J_{\rho \sigma} .
$$

However we cannot proceed further to obtain the full set of the commutation relations as in the three-dimensional case. This is because, using $\left[p^{\mu}, x^{\nu}\right]=-i \eta^{\mu \nu}+o\left(l_{P}\right)$, we obtain

$$
\left[x^{\mu},\left[x^{\nu}, x^{\rho}\right]\right]=-l_{P}^{2} \varepsilon^{\mu \nu \rho \sigma} x_{\sigma}+o\left(l_{P}^{2}\right),
$$

and the Jacobi identity should be violated in the four-dimensional case.

Another possibility of representing the uncertainty relation is

$$
\left[x^{\mu}, p^{\nu}\right]=i \eta^{\mu \nu}+l_{P}^{2} \varepsilon^{\mu \nu \rho \sigma} p_{\rho} p_{\sigma} .
$$

However, since $\left[p^{\mu}, p^{\nu}\right]=o(1)$, the last term would be smaller than the order of $l_{P}^{2}$, and can not work as a realization of the uncertainty relation.

The violation of the Jacobi identity is not a special thing mathematically, and is usual when the algebra is not associative [25]. However, the associativity seems to be deeply embedded in the formulation of quantum mechanics. Before going further, we might have to know the physical reason why we should abandon the associativity. Without any physical motivations, we would have another possibility using some triple product $[a, b, c]$ to represent the uncertainty relation in a form $\varepsilon_{\mu \nu \rho \sigma}\left[x^{\mu}, x^{\nu}, x^{\rho}\right]=l_{P}^{2} x_{\sigma}$.

\section{Summary and discussions}

In this paper, we have investigated a Lorentz covariant formulation of a space-time uncertainty relation. The two uncertainty relations at $\delta t \gtrsim l_{P}$ and $\delta t \lesssim l_{P}$ derived from a thought experiment are combined into a single inequality. We find a minimal area in the four-dimensional 
case, while we find a minimal length in three dimensions. This can be obviously generalized to any space-time dimension $D$, in which a $(D-2)$-volume has a lower bound.

We also discussed a free field theory on a non-commutative space-time, which realizes the space-time uncertainty relation above. We have obtained a representation of the coordinate operators and a positive-definite norm in the momentum representation. The momentum space is two-fold degenerate, and a further investigation would be needed to clarify its origin. We have shown that there exists a mass upper-bound. The existence of the mass upperbound is an expected property of three-dimensional gravity. It is known that a point-particle generates a conical singularity with a deficit angle proportional to its mass [26]. Since a deficit angle cannot exceed $2 \pi$, a mass should have an upper bound. In four dimensions, we seem to be forced to use an unusual algebra to represent the uncertainty relation.

Our space-time uncertainty relation does not respect the parity invariance. Since this invariance is not respected also in the standard model, the violation at the fundamental geometrical level would be interesting rather than disappointing.

The three-dimensional non-commutative space-time seems to be consistently formulated. It would be highly interesting to construct a quantum field theory on this non-commutative space-time and investigate its thermodynamics to compare with the intuitive arguments in our previous paper [15]. We hope we can give some results in this direction in our future works.

\section{Acknowledgments}

The author would like to thank T. Yoneya and H. Suzuki for valuable suggestions, and D.V. Ahluwalia, A. Kempf, M. Li and Y.J. Ng for interesting communication. He is also grateful for the hospitality at Summer Institute '99, Yamanashi, Japan, where this work was initiated. He was supported in part by Grant-in-Aid for Scientific Research (\#09640346), and in part by Priority Area: "Supersymmetry and Unified Theory of Elementary Particles" (\#707), from Ministry of Education, Science, Sports and Culture.

\section{References}

[1] C.W. Misner, K.S. Thorne and J.A. Wheeler, Gravitation, San Fransisco: Freeman, 1973.

[2] G. Veneziano, Europhys. Lett. 2 (1986) 199.

D. Gross and P. Mende, Nucl. Phys. B303 (1988) 407. 
D. Amati, M. Ciafaloni and G. Veneziano, Phys. Lett. B216 (1989) 41.

M. Fabbrichesi and G. Veneziano, Phys. Lett. B233 (1989) 135.

K. Konishi, G. Paffuti and P. Provero, Phys. Lett. B234 (1990) 276.

[3] T. Yonoya, Duality and Indeterminacy Principle in String Theory in "Wondering in the Fields", eds. K. Kawarabayashi and A. Ukawa (World Scientific, 1987), p. 419.

T. Yoneya, String Theory and Quantum Gravity in "Quantum String Theory", eds. N. Kawamoto and T. Kugo (Springer, 1988), p. 23.

T. Yoneya, Mod. Phys. Lett. A4 (1989) 1587.

[4] M. Li and T. Yoneya, Phys. Rev. Lett. 78 (1997) 1219.

M. Li and T. Yoneya, hep-th/9806240.

[5] C.-S. Chu, P.-M. Ho and Y.-C. Kao, Phys. Rev. D60 (1999) 126003.

[6] H. Salecker and E. P. Wigner, Phys. Rev. 109 (1958) 571.

[7] F. Karolyhazy, Nuovo Cim. A42 (1966) 390.

[8] Y. J. Ng and H. van Dam, Mod. Phys. Lett. A9 (1994) 335; A10 (1995) 2801.

[9] S. Doplicher, K. Fredenhagen and J.E. Roberts, Phys. Lett. B331 (1994) 39.

D.V. Ahluwalia, Phys. Lett. B339 (1994) 301.

[10] As a review, see L.J. Garay, Mod. Phys. Lett. A10 (1995) 145.

[11] G. Amelino-Camelia, Nature 398 (1999) 216.

[12] R.J. Adler, I.M. Nemenman, J.M. Overduin and D.I. Santiago, gr-qc/9909017.

[13] Y.J. Ng and H. van Dam, gr-qc/9911054.

G. Amelino-Camelia, gr-qc/9910023.

[14] J.D. Barrow, Phys. Rev. D54 (1996) 6563.

[15] N. Sasakura, Prog. Theor. Phys. 102 (1999) 169.

[16] G. 't Hooft, gr-qc/9310026.

L. Susskind, J. Math. Phys. 36 (1995) 6377.

[17] E.E. Flanagan, D. Marolf and R.M. Wald, to appear in Phys. Rev. D, hep-th/9908070. 
[18] R. Bousso, JHEP 9907 (1999) 063503.

[19] R. Brustein and G. Veneziano, hep-th/9912055.

[20] G. Veneziano, Phys. Lett. B454 (1999) 22.

[21] R.M. Wald, General Relativity, The University of Chicago press, 1984.

[22] H.S. Snyder, Phys. Rev. 71 (1947) 38.

[23] M. Maggiore, Phys. Rev. D49 (1994) 5182; Phys. Lett. B319 (1993) 83.

[24] A. Kempf, G. Mangano and R.B. Mann, Phys. Rev. D52 (1995) 1108.

A. Kempf, J. Math. Phys. 35 (1994) 4483; hep-th/9810215;hep-th/9907160.

[25] As an introduction, see, S. Okubo, Introduction to Octonion and Other Non-Associative Algebras in Physics, Cambridge University press, 1995.

[26] S. Deser, R. Jackiw and G. 't Hooft, Annals Phys. 152 (1984) 220. 